PROCEEDINGS OF THE

AMERICAN MATHEMATICAL SOCIETY

Volume 137, Number 4, April 2009, Pages 1175-1178

S 0002-9939(08)09736-0

Article electronically published on November 4, 2008

\title{
A FAMILY OF LOCAL RINGS WITH RATIONAL POINCARÉ SERIES
}

\author{
JUAN ELIAS AND GIUSEPPE VALLA
}

(Communicated by Bernd Ulrich)

\begin{abstract}
In this note we compute the Poincaré series of almost stretched Gorenstein local rings. It turns out that it is rational.
\end{abstract}

\section{INTRODUCTION}

Let $(R, \mathfrak{n})$ be a regular local ring and $k=R / \mathfrak{n}$ its residue field which we assume of characteristic zero.

Given an ideal $I \subseteq \mathfrak{n}^{2}$, a classical problem in commutative algebra is to study the Poincaré series

$$
\mathbb{P}_{A}(z):=\sum_{i \geq 0} \operatorname{dim}_{k} \operatorname{Tor}_{i}^{A}(k, k) z^{i}
$$

of the local ring $(A=R / I, \mathfrak{m}=\mathfrak{n} / I)$. This is the generating function of the sequence of Betti numbers of a minimal free resolution of $k$ over $A$.

Due to the classical conjecture of Serre, the main issue is the rationality of this series. We know by the example of Anick (see 2] ) that this series can be nonrational, but there are relatively few classes of local rings for which the question has been settled. See 3 for a detailed study of these and other relevant related problems in local algebra.

Given a Cohen-Macaulay local $\operatorname{ring} A=R / I$, we say that $A$ is stretched if there exists an Artinian reduction $B$ of $A$ such that the square of its maximal ideal is a principal ideal. Instead, if the square of the maximal ideal of an Artinian reduction is minimally generated by two elements, we say that $A$ is almost stretched. See [11, [9, 5] and 6] for papers concerning these notions.

In [10] J. Sally computed the Poincaré series of a stretched Cohen-Macaulay local ring and obtained, as a corollary, the rationality of the series. It follows that local Gorenstein rings of multiplicity at most five have rational Poincaré series.

In this paper we compute the Poincaré series of an almost stretched Gorenstein local ring, thus exhibiting its rationality. Using this result, we can prove the rationality of the Poincaré series of any Gorenstein local ring of multiplicity at most seven.

Received by the editors March 31, 2008.

2000 Mathematics Subject Classification. Primary 13D40; Secondary 13H10.

The first author was partially supported by MEC-FEDER MTM2007-67493.

The second author was partially supported by MIUR. 
We are not developing new methods for the computation of the Betti numbers of the minimal $A$-free resolution of $k$; rather we show that the structure theorem we proved in [5] for Artinian almost stretched Gorenstein local rings is very much suitable to the computation of $\operatorname{Tor}_{i}^{A}(k, k)$.

In the following, for a local ring $(A, \mathfrak{m}, k:=A / \mathfrak{m})$ of dimension $d$, we denote by $h$ the embedding codimension of $A$, namely the integer $h:=\operatorname{dim}_{k}\left(\mathfrak{m} / \mathfrak{m}^{2}\right)-d$. Recall, see [1, that the multiplicity $e$ of a Cohen-Macaulay local ring $A$ of embedding codimension $h$ satisfies the inequality $e \geq h+1$. Further, in the extremal case $e=h+1$, it is well known that $\mathbb{P}_{A}(z)$ is rational.

The main result of this paper is the following theorem.

Theorem 1.1. Let $A=R / I$ be an almost stretched Gorenstein local ring of dimension d and embedding codimension $h$. Then

$$
\mathbb{P}_{A}(z)=\frac{(1+z)^{d}}{1-h z+z^{2}} .
$$

\section{Proof of the theorem}

Let $J:=\left(a_{1}, \ldots, a_{d}\right)$ be the ideal generated by a minimal reduction of $\mathfrak{m}$, such that $A / J$ is almost stretched and Gorenstein. Since $\left\{a_{1}, \ldots, a_{d}\right\}$ is a regular sequence on $A$, we have by [12, Satz 1 ,

$$
\mathbb{P}_{A}(z)=(1+z)^{d} \mathbb{P}_{A / J}(z) .
$$

Hence we may assume that $(A=R / I, \mathfrak{m}=\mathfrak{n} / I)$ is an Artinian almost stretched Gorenstein local ring of embedding dimension $h$. In this case we proved in [5, Proposition 4.8, that we can find integers $s \geq t+1 \geq 3$ depending on the Hilbert function of $A$, a minimal system of generators $\left\{x_{1}, \ldots, x_{h}\right\}$ of the maximal ideal $\mathfrak{n}$ of $R$ and an element $a \in R$ such that $I$ is generated by the elements

$$
\left\{x_{1} x_{j}\right\}_{j=3, \ldots, h}, \quad\left\{x_{i} x_{j}\right\}_{2 \leq i<j \leq h}, \quad\left\{x_{j}^{2}-x_{1}^{s}\right\}_{j=3, \ldots, h}, \quad x_{2}^{2}-a x_{1} x_{2}-x_{1}^{s-t+1}, \quad x_{1}^{t} x_{2} .
$$

Further ${\overline{x_{1}}}^{s} \in A=R / I$ is the generator of the socle $0: \mathfrak{m}$ of $A$. Hence

$$
\frac{A}{(0: \mathfrak{m})} \simeq \frac{R}{\left(I+\left(x_{1}^{s}\right)\right)}=\frac{R}{K}
$$

where $K$ is the ideal in $R$ generated by

$$
\left\{x_{1} x_{j}\right\}_{j=3, \ldots, h}, \quad\left\{x_{i} x_{j}\right\}_{2 \leq i<j \leq h}, \quad\left\{x_{j}^{2}\right\}_{j=3, \ldots, h}, \quad x_{2}^{2}-a x_{1} x_{2}-x_{1}^{s-t+1}, \quad x_{1}^{t} x_{2}, \quad x_{1}^{s} .
$$

Notice that by 8 ] we have

$$
\mathbb{P}_{A}(z)=\frac{\mathbb{P}_{A /(0: \mathfrak{m})}(z)}{1+z^{2} \mathbb{P}_{A /(0: \mathfrak{m})}(z)}=\frac{\mathbb{P}_{R / K}(z)}{1+z^{2} \mathbb{P}_{R / K}(z)}
$$

so that we are left to compute the Poincaré series of $R / K$.

It is clear that $\bar{x}_{3}, \ldots, \bar{x}_{h} \in \mathfrak{m} \backslash \mathfrak{m}^{2}$ are elements in the socle of $R / K$. Hence we can compute $h-2$ times [7, Proposition 3.4.4, to get

$$
\mathbb{P}_{R / K}(z)=\frac{\mathbb{P}_{S / L}(z)}{1-(h-2) z \mathbb{P}_{S / L}(z)},
$$

where $S=R /\left(x_{3}, \ldots, x_{h}\right)$ is a two dimensional regular local ring with maximal ideal $\mathfrak{n}=\left(x_{1}, x_{2}\right)$ and $L$ is the ideal

$$
L:=\left(x_{2}^{2}-a x_{1} x_{2}-x_{1}^{s-t+1}, \quad x_{1}^{t} x_{2}, \quad x_{1}^{s}\right) .
$$


From [12, Satz 9, we get

$$
\mathbb{P}_{S / L}(z)=\frac{(1+z)^{2}}{1-3 z^{2}-2 z^{3}}=\frac{1}{1-2 z}
$$

and thus from (2),

$$
\mathbb{P}_{R / K}(z)=\frac{1}{1-h z}
$$

Finally, by (1) we get

$$
\mathbb{P}_{A}(z)=\frac{1}{1-h z+z^{2}}
$$

and the conclusion follows.

A consequence of the above theorem is the rationality of the Poincaré series of any Gorenstein local ring of multiplicity $e \leq h+4$.

Corollary 2.1. Let $A$ be a Gorenstein local ring of dimension d, multiplicity $e$ and embedding codimension $h$. If $e=h+1, h+2, h+3, h+4$, then $\mathbb{P}_{A}(z)$ is rational. If $A$ is a Gorenstein local ring of multiplicity at most seven, then $\mathbb{P}_{A}(z)$ is rational.

Proof. We need only to consider the case $h+2 \leq e \leq h+4$. Since any Artinian reduction $B$ of $A$ is a Gorenstein local ring with the same multiplicity and the same embedding codimension, the possible Hilbert series of $B$ are

$$
\{1, h, 1\},\{1, h, 1,1\},\{1, h, 2,1\},\{1, h, 1,1,1\} .
$$

This proves that $A$ is either stretched or almost stretched. The conclusion follows by Sally's result and the above theorem.

Let $A$ be a Gorenstein local ring of multiplicity at most seven. As before the possible Hilbert series of any Artinian reduction $B$ of $A$ are

$$
\begin{aligned}
& \{1,5,1\},\{1,4,1,1\},\{1,3,2,1\},\{1,3,1,1,1\} . \\
& \{1,2,3,1\},\{1,2,2,1,1\},\{1,2,1,1,1,1\},\{1,1,1,1,1,1,1\} .
\end{aligned}
$$

But $\{1,2,3,1\}$ is not allowed because Gorenstein in codimension two implies complete intersection. In all the remaining cases $A$ is either stretched or almost stretched and we get the conclusion.

Remark 1. Bøgvad in [4] showed that there exist Artinian Gorenstein local rings of multiplicity 26 with nonrational Poincaré series.

Remark 2. The Hilbert function of an almost stretched Artinian Gorenstein local ring $A$ has the following shape:

$$
\begin{array}{|c|c|c|c|c|c|c|c|c|c|}
n & 0 & 1 & 2 & \ldots & t & t+1 & \ldots & s & s+1 \\
\hline H_{A}(n) & 1 & h & 2 & \ldots & 2 & 1 & \ldots & 1 & 0
\end{array}
$$

for integers $s$ and $t$ such that $s \geq t+1 \geq 3$. On the contrary, the Poincaré series of $A$ is independent from $t$ and $s$.

Remark 3. The Poincaré series of stretched and almost stretched Gorenstein local rings with the same dimension and the same embedding dimension coincide; see [10, Theorem 2. 


\section{REFERENCES}

[1] S. S. Abhyankar, Local rings of high embedding dimension, Amer. J. Math. 89 (1967), 10731077. MR0220723 (36:3775)

[2] D. J. Anick, A counterexample to a conjecture of Serre, Ann. of Math. (2) 115 (1982), no. 1, 1-33. MR644015 (86i:55011a)

[3] L. Avramov, Infinite free resolutions, Six lectures on commutative algebra (Bellaterra, 1996), Progr. Math., vol. 166, Birkhäuser, Basel, 1998, pp. 1-118. MR.1648664 (99m:13022)

[4] R. Bøgvad, Gorenstein rings with transcendental Poincaré-series, Math. Scand. 53 (1983), no. 1, 5-15. MR.733933 (85c:13012)

[5] J. Elias and G. Valla, Structure theorems for certain Gorenstein ideals, Michigan J. of Math. 57 (2008), 269-292.

[6] J. Elias and G. Valla, Isomorphism classes of certain complete intersections, in preparation (2008).

[7] T.H. Gulliksen and G. Levin, Homology of local rings, Queen's Papers in Pure and Applied Math., No. 20, Queen's University, Kingston, Ontario, 1969. MR0262227 (41:6837)

[8] G. Levin and L. Avramov, Factoring out the socle of a Gorenstein ring, J. of Algebra 55 (1978), no. 1, 74-83. MR515760 (80j:13015)

[9] M.E. Rossi and G. Valla, Stretched $\mathfrak{m}$-primary ideals, Beiträge zur Algebra und Geometrie 42 (2001), no. 1, 103-122. MR1824753 (2002d:13023)

[10] J. Sally, The Poincaré series of stretched Cohen-Macaulay rings, Canad. J. Math 32 (1980), no. 5, 1261-1265. MR596109 (82b:13011)

[11] J. Sally, Stretched Gorenstein rings, J. London Math. Soc. 20 (1979), no. 2, 19-26. MR545198 (80k:14006)

[12] G. Scheja, Uber die Bettizahlen lokaler Ringe, Math. Ann. 155 (1964), 155-172. MR0162819 $(29: 123)$

Departament d’Àlgebra i Geometria, Facultat de Matemàtiques, Universitat de Barcelona, Gran Via 585, 08007 Barcelona, Spain

E-mail address: elias@ub.edu

Dipartimento di Matematica, Università di Genova, Via Dodecaneso 35, 16146 Genova, ITALY

E-mail address: valla@dima.unige.it 